\title{
Antibiotic policy of Gastroenterology Surgery Center in Egypt
}

\author{
Noha El-Mashad ${ }^{1}$, Eman Elsayed ${ }^{1}$, Mohamed Elshobary², \\ Yomna Ahmed Setate ${ }^{3}$ and Mohammed Elshaer ${ }^{1 *}$ \\ ${ }^{1}$ Department of Clinical Pathology, Faculty of Medicine, Mansoura, Egypt \\ ${ }^{2}$ Mansoura Gastroenterology Surgical Center, Egypt \\ ${ }^{3}$ Mansoura Specialized Hospital, Egypt \\ *Corresponding author
}

\section{A B S T R A C T}

Antimicrobial resistance is considered a major health threat for patients and healthcare providers. It challenges the control of infectious diseases, jeopardizes any medical progress and imposes huge treatment costs. This study aimed to

\section{Keywords}

Carbapenemase, Drug resistance, Sensitivity tests, Drug resistance, Infection control

Article Info

Accepted:

22 March 2020

Available Online:

10 April 2020 design an effective antibiotic policy for treatment of different types of infections at Gastrointestinal Surgical Center, Mansoura, Egypt in order to reduce antibiotic resistance, and to minimize unnecessary costs. From 1/1/2019 to 31/12/2019, samples were obtained according to the site of infection, cultured on suitable media. Automated identification of isolates was performed by Vitek 2 system whereas antibiotic susceptibility testing was done by disc diffusion method. Isolates with multidrug resistance were also assessed for susceptibility by Vitek 2 system. Out of 3300 microbiologic samples, 1190 (36.1\%) were positive. 862 of $1190(72.4 \%)$ were gram-negative \& 328 of 1190 (27.6\%) gram-positive. The patterns of resistance observed were MRSA (37.1\%), ESBL (8.5\%) and Carbapenemases (8.9\%). An antibiogram was designed for each infection type including the first-line therapy protocol. Antibiotic initiation or change should be done after sending appropriate cultures Once culture reports are available, the physician must step down to the narrowest spectrum antibiotic, the most efficient and cost-effective option.

\section{Introduction}

Antimicrobial resistance is considered a major health threat for patients and healthcare providers. It challenges the control of infectious diseases, jeopardizes any medical progress and imposes huge treatment costs. In the European Union, about 25000 patients die each year from infections caused by multidrug-resistant bacteria and the estimated costs are about 1.5 billion euros per year(1).

The rapid emergence of resistant bacteria is occurring worldwide, endangering the efficacy of antibiotics, which have transformed medicine and saved millions of 
lives. Resistance has emerged even to newer, more potent antimicrobial agents such as carbapenems which can be attributed to the misuse or overuse of antibiotics. Hence the importance of inclusion of the antibiotic policy in the infection control program (2-5).

The antibiotic policy is the set of written guidance that is to assist and support clinicians with decisions regarding the optimal selection, dose and duration of an antimicrobial agent for the treatment of an infectious disease in the hospital. It covers empirical treatment, specific treatment, and also agents for prophylaxis $(6,7)$.

The basic principles are to be direct evidencebased medicine and using local antibiogram. An antibiotic policy is now more necessary than ever for clinical, epidemiological and economic reasons(7).

The Infection Prevention and Control Committee acts as an advisory body to the medical staff, analyzing the epidemiology of the infections, improving the appropriate use of antimicrobials and provide adequate training for healthcare workers(8).

The agreement of hundreds of professionals on indications, dosage and duration of antibiotic treatment, based on the best scientific evidence and local guides is complex, but it can be done. The key to this is that the Infection Committee develops antimicrobial policy through a multidisciplinary team and professional leadership, and has the institutional support to ensure that the proper use of antimicrobials (8).

This study aimed to design an effective antibiotic policy for treatment of different types of infections at Gastrointestinal Surgical Center, Mansoura, Egypt to reduce antibiotic resistance, and to minimize unnecessary costs.

\section{Materials and Methods}

This study included 3300 different samples from patients admitted to Gastrointestinal Surgery Center (GISC), Mansoura University, Egypt in the period from 1/1/2019 to $31 / 12 / 2019$, with different signs and symptoms of infections. We classified GISC into 5 areas (according to the patients' distribution) including:

Liver transplantation unit on the 7th floor Patient admission wards on the $5^{\text {th }} \& 6^{\text {th }}$ floors Transplantation ICU on the 2 nd floor

Surgical ICU on the 2nd floor

Outpatient clinics on the ground floor

Microbiological samples were collected according to the site of infection. Different sample types included blood, urine, sputum, wound swab, drain aspirate, peritoneal fluid aspirate, and throat swab.

Cultures on the suitable media were done in the microbiology laboratory. Culture media used included blood agar, MacConkey agar, SS agar. All cultures were carried out by significant colony count.

Automated identification of isolates was performed by Vitek 2 system whereas antibiotic susceptibility testing was done by disc diffusion method followed CLSI - 2012 guidelines. Isolates with multidrug resistance were also assessed for susceptibility by Vitek 2 system (9). Anaerobic culture is not a routine work in our laboratory and performed only by request, therefore the results were excluded.

\section{Results and Discussion}

Out of 3300 microbiologic samples, 1190 $(36.1 \%)$ were positive. 862 of $1190(72.4 \%)$ were gram negative \& 328 of $1190(27.6 \%)$ gram-positive. The distribution of different samples obtained in GISC illustrated in Table 
1. Most of the cultures were isolated from surgical drains $(47.4 \%)$, urine $(17.18 \%)$ and sputum $(14.7 \%)$.

Table 2 presents the frequency of aerobic bacterial growth in each hospital ward; the majority was obtained from surgical wards (33.8\%) followed by Surgical ICU (25.3\%) and Outpatient Clinic (15.5\%).

In Table 3, the majority of gram-negative bacteria were obtained from surgical drains $(60.6 \%)$ while the majority of gram-positive bacteria were obtained from Sputum (26.8\%).

The gram-negative bacteria obtained in our study were classified according to the infected site, as illustrated in Table 4.

The patterns of resistance observed were
MRSA (37.1\%), ESBL (8.5\%) and Carbapenemases (8.9\%) (Table 5).

The antibiogram obtained for each infection type is presented in Tables 6-9, whereas the first line therapy protocol is illustrated in Table 10.

The following antibiotics were reserved for use in resistant cases

Linezolid (Oxazolidinone).

Carbapenems (recently introduced types).

Moxifloxacin $\quad\left(4^{\text {th }} \quad\right.$ generation fluoroquinolone).

Tigecycline (Glycylcyclines).

Table.1 Frequency of different samples in GISC

\begin{tabular}{|c|c|c|c|c|c|c|}
\hline & \multicolumn{6}{|c|}{ Total prevalence in all samples } \\
\hline & \multicolumn{2}{|c|}{ Total cultured } & \multicolumn{2}{|c|}{ Growth } & \multicolumn{2}{|c|}{ No growth } \\
\hline & $\mathbf{N}$ & $\%$ & $\mathbf{N}$ & $\%$ & $\mathbf{N}$ & $\%$ \\
\hline Blood & 317 & 9.6 & 105 & 8.8 & 212 & 10.0 \\
\hline Urine & 586 & 17.8 & 205 & 17.2 & 381 & 18.1 \\
\hline Sputum & 485 & 14.7 & 187 & 15.7 & 298 & 14.1 \\
\hline Wound & 237 & 7.2 & 79 & 6.6 & 158 & 7.5 \\
\hline Drain & 1565 & 47.4 & 578 & 48.6 & 987 & 46.8 \\
\hline Peritoneal & 27 & 0.8 & 9 & 0.8 & 18 & 0.9 \\
\hline Throat & 83 & 2.5 & 27 & 2.3 & 56 & 2.7 \\
\hline Total & 3300 & 100 & 1190 & 100 & 2110 & 100.0 \\
\hline
\end{tabular}


Table.2 Frequency of aerobic bacterial growth in each ward in GISC

\begin{tabular}{|l|l|c|c|c|c|c|}
\hline & & \multicolumn{2}{|c|}{$\begin{array}{c}\text { Gram } \\
\text { negative }\end{array}$} & \multicolumn{2}{c|}{$\begin{array}{c}\text { Gram } \\
\text { positive }\end{array}$} & Total \\
\cline { 3 - 7 } & & N & $\%$ & N & $\%$ & \\
\hline O.C & Floors & 134 & 15.5 & 53 & 16.2 & 187 \\
\hline \multirow{2}{*}{ Wards } & 291 & 33.8 & 114 & 34.8 & 405 \\
\hline \multirow{2}{*}{ Transplantation } & ICU-Surgical & 218 & 25.3 & 79 & 24.1 & 297 \\
\hline \multirow{2}{*}{ Total } & Transplantation & 108 & 12.5 & 43 & 13.1 & 151 \\
\cline { 2 - 7 } & ICU-Transplant & 111 & 12.9 & 39 & 11.9 & 150 \\
\hline
\end{tabular}

Table.3 Distribution of microorganisms in each sample type in GISC

\begin{tabular}{|l|c|c|c|c|c|}
\hline \multirow{2}{*}{ Blood } & \multicolumn{2}{|c|}{ Gram negative } & \multicolumn{2}{c|}{ Gram positive } & \multirow{2}{*}{ Total } \\
\cline { 2 - 5 } & $\mathbf{N}$ & $\mathbf{\%}$ & $\mathbf{N}$ & $\mathbf{\%}$ & \\
\hline Urine & 54 & 6.3 & 51 & 15.5 & 105 \\
\hline Sputum & 127 & 14.7 & 78 & 23.8 & 205 \\
\hline Wound & 99 & 11.5 & 88 & 26.8 & 187 \\
\hline Drain & 42 & 4.9 & 37 & 11.3 & 79 \\
\hline Peritoneal & 522 & 60.6 & 56 & 17.1 & 578 \\
\hline Throat & 9 & 1.0 & 0 & 0 & 9 \\
\hline Total & 9 & 1.0 & 18 & 5.5 & 27 \\
\hline
\end{tabular}

Table.4 Frequency of aerobic bacterial growth in each ward in GISC

\begin{tabular}{|l|l|c|c|c|c|c|}
\hline & & \multicolumn{2}{|c|}{$\begin{array}{c}\text { Gram } \\
\text { negative }\end{array}$} & \multicolumn{2}{c|}{$\begin{array}{c}\text { Gram } \\
\text { positive }\end{array}$} & Total \\
\cline { 1 - 6 } & & N & $\%$ & N & $\%$ & \\
\hline O.C & & 134 & 15.5 & 53 & 16.2 & 187 \\
\hline Surgical wards & Floors & 291 & 33.8 & 114 & 34.8 & 405 \\
\cline { 2 - 6 } & ICU & 218 & 25.3 & 79 & 24.1 & 297 \\
\hline \multirow{2}{*}{ Transplantation } & Transplantation & 108 & 12.5 & 43 & 13.1 & 151 \\
\hline & ICU & 111 & 12.9 & 39 & 11.9 & 150 \\
\hline Total & & 862 & 100 & 328 & 100 & 1190 \\
\hline
\end{tabular}


Table.5 Patterns of resistance

\begin{tabular}{|l|c|c|c|}
\hline & N & $\%$ & \\
\hline MRSA & $65 / 175$ & 37.1 & Out of Staph aureus \\
\hline ESBL & $73 / 862$ & 8.5 & Out of gram negative bacteria \\
\hline Carbapenem resistance & $106 / 1190$ & 8.9 & Out of all culture growths \\
\hline
\end{tabular}

Table.6 Blood stream infection-antibiogram

\begin{tabular}{|c|c|c|}
\hline $\begin{array}{l}\text { Most common } \\
\text { pathogens }\end{array}$ & $\begin{array}{l}\text { Prevalence } \\
\quad(\%)\end{array}$ & Antibiotic Sensitivity (\%) \\
\hline Staph aureus & 33.3 & $\begin{array}{l}\text { Ampicillin/Sulbactam } 66.7 \% \text {, Piperacillin/Tazobactam } \\
66.7 \% \text {, Ampicillin } 33.3 \%\end{array}$ \\
\hline E.coli & 22.2 & Ciprofloxacin $100 \%$, Cefoxitin $100 \%$, Cefepime $50 \%$ \\
\hline Citrobacter & 11.1 & $\begin{array}{l}\text { Gentamycin 100\%, Ampicillin/Sulbactam 100\%, } \\
\text { Amikacin 100\%, Imipenem 100\%, }\end{array}$ \\
\hline Serratia & 22.2 & Doxycycline $100 \%$, Imipenem $100 \%$ \\
\hline $\begin{array}{l}\text { Other Gram } \\
\text {-ve bacilli }\end{array}$ & 11.1 & $\begin{array}{l}\text { Levofloxacin } 100 \% \text {, Doxycycline } 100 \% \text {, Imipenem } \\
\text { 100\%, Piperacillin/Tazobactam 100\% }\end{array}$ \\
\hline
\end{tabular}

Table.7 Urinary tract infection-antibiogram

\begin{tabular}{|c|c|c|}
\hline $\begin{array}{l}\text { Most common } \\
\text { pathogens }\end{array}$ & $\begin{array}{l}\text { Prevalence } \\
(\%)\end{array}$ & Antibiotic Sensitivity (\%) \\
\hline $\begin{array}{l}\text { Coagulase -ve } \\
\text { staph }\end{array}$ & 10.5 & $\begin{array}{l}\text { Levofloxacin 50\%, Clindamycin 50\%, Doxycycline } \\
50 \% \text {, Amikacin 50\% }\end{array}$ \\
\hline Staph aureus & 5.3 & $\begin{array}{l}\text { Nitrofurantoin } 100 \% \text { Imipenem } 100 \% \text {. Levofloxacin } \\
50 \% \text {, Clindamycin } 50 \% \text {, Doxycycline } 50 \% \text {, }\end{array}$ \\
\hline E coli & 21.1 & $\begin{array}{l}\text { Ampicillin 25\%, Gentamicin 25\%, Amikacin 25\%, } \\
\text { Piperacillin/Tazobactam } 25 \% \text {, }\end{array}$ \\
\hline Klebsiella & 10.5 & $\begin{array}{l}\text { Cefoxitin } 50 \% \text {, Gentamycin 50\%, Ciprofloxacin 50\%, } \\
\text { Levofloxacin 50\%, Ceftazidime 50\%, Cefepime 50\%, } \\
\text { Cefoperazone/Sulbactam 50\%, } \\
\text { Piperacillin/Tazobactam50\%, }\end{array}$ \\
\hline Citrobacter & 5.3 & $\begin{array}{l}\text { Amikacin 100\%, Imipenem 100\%, Cefoperazone/ } \\
\text { Sulbactam 50\%, Piperacillin/Tazobactam50\%, } \\
\text { Gentamicin 50\%, Ciprofloxacin 50\%, }\end{array}$ \\
\hline Proteus & 5.3 & $\begin{array}{l}\text { Ceftriaxone } 100 \% \text {, Gentamycin } 100 \% \text {, Ciprofloxacin } \\
100 \% \text {, Ceftazidime } 100 \%\end{array}$ \\
\hline Salmonella & 5.3 & $\begin{array}{l}\text { Levofloxacin 100\%, Piperacillin/Tazobactam100\%, } \\
\text { Imipenem 100\%, Cefoperazone/Sulbactam 100\%, }\end{array}$ \\
\hline
\end{tabular}


Table.8 Sputum culture - antibiogram

\begin{tabular}{|c|c|c|}
\hline $\begin{array}{l}\text { Most common } \\
\text { pathogens }\end{array}$ & $\begin{array}{l}\text { Prevalence } \\
\qquad(\%)\end{array}$ & Antibiotic Sensitivity (\%) \\
\hline $\begin{array}{l}\text { Coagulase -ve } \\
\text { staph }\end{array}$ & 18.8 & $\begin{array}{l}\text { Cefoperazone/Sulbactam 100\%, Piperacillin } 100 \% \text {, } \\
\text { Ampicillin/Sulbactam } 66.7 \% \text {, Doxycycline } 66.7 \% \text {, } \\
\text { Levofloxacin } 33.3 \% \text {, Erythromycin } 33.3 \% \text {, Cefoxitin } \\
33.3 \% \text {, }\end{array}$ \\
\hline $\begin{array}{l}\text { Staphylococcus } \\
\text { aureus }\end{array}$ & 12.5 & $\begin{array}{l}\text { Erythromycin } 100 \% \text {, Ampicillin/Sulbactam 50\%, } \\
\text { Doxycycline 50\%, Piperacillin/Tazobactam50\% }\end{array}$ \\
\hline E. coli & 6.3 & $\begin{array}{l}\text { Amikacin } 100 \% \text {, Piperacillin/Tazobactam } 100 \% \text {, } \\
\text { Cefoperazone/Sulbactam } 100 \% \text {, Doxycycline } 100 \% \text {, } \\
\text { Cefoxitin } 100 \%\end{array}$ \\
\hline Klebsiella & 25.0 & $\begin{array}{l}\text { Piperacillin/Tazobactam50\%, Ciprofloxacin } 25 \% \text {, } \\
\text { Clindamycin } 25 \% \text {, }\end{array}$ \\
\hline Citrobacter & 12.5 & $\begin{array}{l}\text { Piperacillin/Tazobactam } 100 \% \text {, Ciprofloxacin } 50 \% \text {, } \\
\text { Erythromycin } 50 \% \text {, }\end{array}$ \\
\hline Serratia & 6.3 & $\begin{array}{l}\text { Ampicillin/Sulbactam } 100 \% \text {, } \\
\text { Piperacillin/Tazobactam } 100 \% \text {, Ceftriaxone } 100 \% \text {, } \\
\text { Ciprofloxacin } 100 \% \text {, }\end{array}$ \\
\hline Salmonella & 18.8 & Imipenem $33.3 \%$, Cefoperazone $33.3 \%$ \\
\hline
\end{tabular}

Table.9 Wound culture - antibiogram

\begin{tabular}{|c|c|c|}
\hline $\begin{array}{l}\text { Most common } \\
\text { pathogens }\end{array}$ & $\begin{array}{l}\text { Prevalence } \\
\qquad(\%)\end{array}$ & Antibiotic Sensitivity (\%) \\
\hline $\begin{array}{l}\text { Staphylococcus } \\
\text { aureus }\end{array}$ & 28.6 & $\begin{array}{l}\text { Piperacillin/Tazobactam } 100 \% \text {, Ciprofloxacin 50\%, } \\
\text { Erythromycin 50\%, Vancomycin 100\%, Piperacillin } 50 \%\end{array}$ \\
\hline Pseudomonas & 42.9 & $\begin{array}{l}\text { Doxycycline } 100 \% \text {, Gentamicin } 33.3 \% \text {, Imipenem } 33.3 \% \text {, } \\
\text { Cefoperazone/Sulbactam } 33.3 \% \text {, Clindamycin } 33.3 \% \text {, }\end{array}$ \\
\hline Serratia & 14.3 & $\begin{array}{l}\text { Imipenem } 100 \%, \text { Cefoperazone/Sulbactam } 100 \% \text {, } \\
\text { Doxycycline } 100 \%\end{array}$ \\
\hline $\begin{array}{l}\text { Other Gram } \\
\text {-ve bacilli }\end{array}$ & 14.3 & $\begin{array}{l}\text { Imipenem } 100 \% \text {, Doxycycline } 100 \% \text {, } \\
\text { Cefoperazone/Sulbactam 50\%, Clindamycin } 50 \%\end{array}$ \\
\hline
\end{tabular}


Table.10 First line therapy protocol at GISC

\begin{tabular}{|l|l|}
\hline Blood stream infection & $\begin{array}{l}\text { Ampicillin/Sulbactam,Imipenem, } \\
\text { Piperacillin/Tazobactam, Ciprofloxacin, } \\
\text { Ceftazidime }\end{array}$ \\
\hline Respiratory Tract Infection & $\begin{array}{l}\text { Ampicillin/Sulbactam,Cefoxitin, } \\
\text { Piperacillin/Tazobactam }\end{array}$ \\
\hline Wound Infection & $\begin{array}{l}\text { Piperacillin/Tazobactam,Imipenem,Erythromycin, } \\
\text { Clindamycin }\end{array}$ \\
\hline Urinary Tract Infections (UTI) & Levofloxacin, Doxycycline, Clindamycin \\
\hline
\end{tabular}

Antibiotic resistance may occur even with the proper use of antibiotics, widespread and inappropriate use of antibiotics makes the situation even worse. In more developed countries, there are a number of contributing factors, such as over-the-counter antibiotics, poor patient compliance, and inappropriate selection of antibiotics and overprescribing.

The present study was limited by its retrospective design, important clinical conditions, previous administration of antibiotics and their duration and dosage were not available for analysis. Nonetheless, the strength of this study can be explained by its local nature, which reflects the magnitude of the problem of bacterial resistance in a single center in Egypt.

To our knowledge, this report is the first to instruct an antibiotic policy in single center in Egypt which may have important findings for practicing physicians and authorities involved in hospital formulary in the region regarding empirical antibiotic selection and utilization.

Conclusions are as follows:

Antibiotic initiation must be done after sending appropriate cultures

Choosing antibiotic therapy should follow the hospital policy whenever possible. If alternatives are chosen, the clinician must document the reason in the case records.

The need for antimicrobial therapy should be reviewed on a daily basis. For most infections, $5-7$ days of antimicrobial therapy is sufficient.

All IV antibiotics may only be given for 48 72 hours, then switch to oral antibiotic(s) or switch to an IV narrow spectrum alternative.

Once culture reports are available, the physician shall step down to the narrowest spectrum antibiotic, the most efficient and most cost-effective option.

\section{References}

1. ECDC/EMEA Joint Working Group. ECDC/ EMEA Joint Technical Report: The bacterial challenge: time to react. Stockholm: European Centre for Disease Prevention and Control; 2009. Available at:http://www.ecdc.europa.eu/en/publica tions/Publications/Forms/ECDC_DispFo rm.aspx?ID=444. Accessed April 8, 2018.

2. Gould IM, and Bal AM. New antibiotic agents in the pipeline and how they can overcome microbial resistance. Virulence. 2013; 4(2): 185-191.

3. Sengupta S, Chattopadhyay MK, Grossart HP. The multifaceted roles of antibiotics and antibiotic resistance in nature. Front Microbiol. 2013; 4: 47.

4. Wright GD. Something new: revisiting natural products in antibiotic drug 
discovery. Can J Microbiol. 2014; 60(3): 147-154.

5. Golkar Z, Bagazra O, Pace DG. Bacteriophage therapy: a potential solution for the antibiotic resistance crisis. J Infect Dev Countries. 2014; 8(2):129-136.

6. MacDougall $\mathrm{C}$, and Polk RE. Antimicrobial Stewardship Programs in Health Care Systems. Clin Microbiol Rev. 2005; 18(4): 638-656.

7. Cisneros JM, Pérez-Moreno MA, GilNavarro MV. The antibiotic policy. The Infection Committee and antimicrobial use. Infec Microbiol Clin., 2014; 32(8):
533-536.

8. Best Practices for Infection Prevention and Control Programs in Ontario In All Health Care Settings: Provincial Infectious Diseases Advisory Committee (PIDAC). (3rd edition). 2012. Available at: https://www.publichealthontario. ca/en/.../BP_IPAC_Ontario_HCSettings _2012.pdf. Accessed March 20, 2019.

9. CLSI Clinical and Laboratory Standards. Wayne, Pennsylvania: Performance standards for antimicrobial susceptibility testing (M100eS22). Clinical and Laboratory Standards Institute; 2012.

\section{How to cite this article:}

Noha El-Mashad, Eman Elsayed, Mohamed Elshobary, Yomna Ahmed Setate and Mohammed Elshaer. 2020. Antibiotic policy of Gastroenterology Surgery Center in Egypt. Int.J.Curr.Microbiol.App.Sci. 9(04): 2748-2755. doi: https://doi.org/10.20546/ijcmas.2020.904.324 